\title{
Mediatisierte sexualisierte Gewalt erkennen: A_Rendelle \& Dior
}

„Die Frage ist ja gerade, wenn man im Nachhinein so ein Chat-Protokoll liest, ne, dann denkt man, ja klar, war ja am Anfang schon völlig klar." (Psychologe, spezialisierte Fachberatung, Focus Group Interviews).

- Zusammenfassung Ziel der Falldiskussion ist eine Sensibilisierung für mediatisierte sexualisierte Gewalt. Dazu werden entlang eines Chatauszugs Maßstäbe der fachlichen Bewertung ausgelegt. Ein Transfer ist auch in Zusammenhänge möglich, in denen Hinweise auf Gewalt nicht in Chatverläufen, sondern in Foto-, Video- und Tonaufnahmen enthalten sind. Das Kapitel bespricht somit fachliche Grundlagen, die über den konkreten Fall hinausweisen. Die Ausschnitte des Chats verzichten auf Kontextinformationen zu den Chattenden mit den Nicknamen A_Rendelle und Dior, um unmittelbare Assoziationen durch die Kommunikation auszulösen. Eine ausführliche Reflexion sowie die Fortsetzung des Chats schließen an.

\section{Fallvignette $\mathrm{A} \_$Rendelle und Dior}

\section{Chatverlauf Teil 1, 22. August}

15:43 A_Rendelle: Eyyyy!!! Du bist ja online! Ich dachte, du hast so viel zu tun?!

15:44 Dior:

Sollte ich auch. Aber früher Feierabend gemacht.

15:44 A_Rendelle: Wieso?

15:46 Dior: Zu kompliziert jetzt! Wie war dein Tag, Schatz? 
15:46 A_Rendelle: Joa. Lehrer nerven. Dies das. Bla. ABER nach der Schule hat es so geschüttet. Da „musste“ ich mir den Schirm von einem anderen Mädchen mopsen. Ups. XD

15:47 Dior: Wie das Mopsen so die Möpse. Ups. ;-)

15:47 A_Rendelle: Hihi. Ups.

15:47 Dior:

Meine ich aber ernst. Sind wirklich schon sehr schön deine Knospen.

15:48 A_Rendelle: Dankeschön, Schatz. Aber woher willst du das so genau wissen??

15:48 Dior: Gutes Auge. Erkenne eine echte Frau auf zwei Kilometer Entfernung. Zum Beispiel das Bild vor der Brücke. Yamyam!

15:48 A_Rendelle: Hihi. Danke. Aber da erkennt man doch gar nix.

15:49 Dior: $\quad$ Stimmt. Jetzt wo du es sagst ... Dann schick mal ein richtiges Bild! :-)

15:55 A_Rendelle: Würde dich viel lieber bei mir haben und richtig tief spüren.

\section{Reflexionsfragen}

- Was nehmen wir im Chat wahr?

- Was löst der Chat in uns aus?

- Wie stehen wir zu den Chattenden und ihrem Verhalten?

- Wie kann die Situation fachlich eingeordnet werden?

\subsection{Reflexionen zur fachlichen Einordnung des Fallbeispiels (Teil 1)}

Eine Verschleierung von Gewaltmotiven und sexualisierten Absichten kennzeichnet Täter*innenstrategien im Allgemeinen. Daher ist sexualisierte Gewalt für Betroffene und deren soziales Umfeld nicht immer eindeutig erkennbar bzw. offensichtlich. In mediatisierten Kontexten gilt dies besonders aufgrund digitaler Elemente der Kommunikation. Hinzu kommen Unsicherheiten seitens Erwachsener mit Blick auf das, was ,normale“ Jugendkultur - etwa den gleichberechtigten Austausch von sexuellen Inhalten unter Peers - von sexualisierter Gewalt unterscheidet. Selbst bei Expert*innen löst mediatisierte sexualisierte Gewalt Ratlosigkeit und Verwirrung aus (Kärgel \& Vobbe, 2020). Wichtig sind 
deswegen fachliche Maßstäbe, eine Sensibilisierung und eine dahingehende Haltung, um Hinweise auf Gewaltdynamiken erkennen, adäquat einordnen und sich positionieren zu können. Die Entwicklung einer Haltung setzt die Beschäftigung mit eigenen einstellungsbasierten Bewertungsmustern voraus (Albrecht, 2017). Unsere Bewertungsmuster bemerken wir mitunter an Assoziationen und Gefühlen, die der Chat oder die nachfolgende Reflexion in uns auslöst. Widerstände oder alternative fachliche Einschätzungen zu den Ausführungen betrachten wir als Anlass für eine Auseinandersetzungskultur und nicht als Beleg für einen Irrtum.

\subsubsection{Was nehmen wir wahr?}

Die Situation entsteht im Chat zweier Personen in einem Messenger. Die Dauer und Art des Verhältnisses oder eine Altersdifferenz zwischen den Chattenden kann nicht bestimmt werden. Wir identifizieren A_Rendelle durch die Körperund Geschlechtsbezüge Diors (,echte Frau“) als weiblich. Des Weiteren nehmen wir an, dass sie aufgrund ihrer Sprache, der Bezugnahme auf Schule und die Formulierung ,von einem anderen Mädchen“ noch ein Kind oder eine Jugendliche ist. Hinweise auf Diors Geschlecht und Alter bleiben assoziativ. Die Chattenden positionieren sich als Liebespaar. Sie greifen auf Formulierungen und Floskeln zurück (,Schatz"), die genauso der Kommunikation in langjährigen Partnerschaften von Erwachsenen entstammen könnten. Zugleich ist eine solche Inszenierung von Rollenmodellen zwischen jungen Menschen in digitalen Kontexten nicht völlig unüblich (Scarcelli, 2015). In den im HUMAN-Projekt durchgeführten Focus Group Interviews äußern die Expert*innen dennoch Skepsis, dass zwischen den Chattenden eine längere Beziehung bestünde. Diors Bewertungen von A_Rendelles Körper scheinen sich auf Fotos zu beschränken (,Erkenne eine echte Frau auf zwei Kilometer Entfernung. Zum Beispiel das Bild vor der Brücke."). Offenbar hat noch kein Kontakt jenseits digitaler Medien stattgefunden.

„Ich muss sagen, gleichzeitig empfinde ich auch eine gewisse Verwirrung, weil aus diesem Teil des Chats entnehme ich erstmal, ,hier Dankeschön, Schatz', also eine Nähe, wir sind irgendwie total vertraut bis intim miteinander. ,Aber woher willst du das so genau wissen', das heißt, ,du hast', Subtext für mich, ,du hast mich ja noch gar nicht in natura gesehen. Wir kennen uns ja nur über online, oder du hast mich in den Fotos gesehen. ““

Psychologe, spezialisierte Fachberatung, FGI

$\mathrm{Ab}$ 15:47 im Chatausschnitt sexualisiert Dior die Kommunikation mit A_Rendelle aktiv. Als Sexualisierung sind Verhaltensweisen und Darstellungen zu 
verstehen, mit denen sexuelle gegenüber anderen Situationsmerkmalen hervorgehoben werden, mit anderen Worten eine Atmosphäre oder Kommunikation sexuell aufgeladen wird (Orbach, 2013). Eine Sexualisierung kann mitunter durch die beabsichtigte Mehrdeutigkeit von Kommentaren erzielt werden. So kontert Dior Äußerungen A_Rendelles, um A_Rendelles Körper, genauer ihre Brüste, zu thematisieren (,wie das Mopsen so die Möpse"). Es geht anschließend vorwiegend um A_Rendelles Körper. Dior stellt hierbei Zusammenhänge zu Weiblichkeitsvorstellungen her (,echte Frau“), die A_Rendelle aufwerten sollen. Zwar hinterfragt A_Rendelle die Bewertungsmaßstäbe Diors (,,woher willst du das so genau wissen“), doch nimmt Dior A_Rendelles Rückfrage zum Anlass, ein „richtiges Bild“ zu fordern. Aus dem sexualisierten Situationskontext ergibt sich, dass auf dem geforderten Foto mehr zu erkennen sein müsse, es sich also um ein sexualisiertes Foto oder Nacktfoto handeln müsse.

Unklar ist, ob der sechsminütigen Pause zwischen Diors Forderung nach einem Foto und A_Rendelles Antwort eine Bedeutung beigemessen werden sollte. Die Stelle eignet sich als Beispiel für die Auseinandersetzung mit eigenen einstellungsbasierten Bewertungsmustern. Gehen wir davon aus, dass A_Rendelle zögert, und falls ja, wie kommen wir im gegebenen Zusammenhang zu der Vermutung? Oder halten wir die Pause für irrelevant, weil sie unterschiedlichste Gründe haben kann und nur im sonstigen Gesprächsrhythmus auffällt?

A_Rendelles Äußerung, sie würde Dior viel lieber bei sich haben und richtig tief spüren, wurde in den Focus Group Interviews dagegen als Ambivalenz und Bewältigungsversuch eines potenziellen Kontrollverlusts diskutiert. Diesbezügliche Deutungen der Expert*innen reichen von:

„Ist das nicht so eine etwas ambivalente Formulierung mindestens? Richtig tief spüren, kann sich ja wirklich so, also so ganz intensiv, so persönlicher Austausch und sich ganz nah sein. Aber auch die andere Assoziation ist, dass man denkt, es geht um Penetration, ne?"

Psychologe, spezialisierte Fachberatung, FGI

bis:

„Es gibt ein diffuses Gefühl von ,irgendwas ist hier nicht ganz in Ordnung. Aber ich will eigentlich auch die Beziehung und was muss ich denn dafür geben?` Es ist anscheinend ein Quidproquo, ich kriege Aufmerksamkeit, wenn ich sexuell mitmache. $\mathrm{Zu}$ explizit, ist mir auch zu krass. Abgrenzen geht nicht, mh, was ist, wenn ich jetzt dadurch die Beziehung verliere?“"

Psychologin, Täter*innentherapie, FGI 


\title{
4.1.2 Welche Hinweise auf mögliche mediatisierte sexualisierte Gewalt enthält der Chat?
}

Die im HUMAN-Projekt erhobenen Falldokumentationen und Interviews mit Gewaltbetroffenen zeigen, dass es oft Erziehungsberechtigte oder Pädagog*innen sind, die zufällig mit sexualisierten Online-Interaktionen in Kontakt kommen, in die Kinder und Jugendliche verwickelt sind. Erwachsene verschaffen sich nach anfänglichen Hinweisen und Vermutungen auch gezielt Zugang zu mobilen Endgeräten junger Menschen.

\begin{abstract}
„Wir [Anmerkung: Kindesmutter und Tochter] sind halt fast zehn Jahre jetzt mittlerweile alleine. Und ich wollte mit ihr über diesen WhatsApp-Kontakt mit ihrem Vater sprechen und habe dabei den Kontakt zu diesem Chat mit diesem Fritz wiedergefunden.“
\end{abstract}

Mutter einer Gewaltbetroffenen

„Der KV hat die Mails/Bilder auf dem Handy von Eva gesehen, als er einen Blick darauf geworfen hatte, um zu kontrollieren, was seine Tochter im Internet macht.“

Falldokumentation 4.1

Somit sind es in erster Linie Menschen des sozialen Umfeldes junger Menschen, die sich an Helfende wenden, um Unterstützung bei der Einordnung mediatisierter sexualisierter Interaktionen zu erhalten. ${ }^{1}$ In der Praxis gehen damit meist weitere Eindrücke einher, beispielsweise zum Alter, zur Lebenssituation und dem Reifestand. Eine in der Eigenschaft digitaler Kommunikation begründete Uneindeutigkeit (Diffusion) bleibt trotzdem in vielen Fällen eine Herausforderung bei der Bewertung mediatisierter sexualisierter Gewalt. Das bemerken auch Expert*innen in den Focus Group Interviews.

„Die Frage ist ja gerade, wenn man im Nachhinein so ein Chat-Protokoll liest, ne, dann denkt man, ja klar, war ja am Anfang schon völlig klar, ne. Aber was sagst du, wenn man nur den Anfang hat.“

Psychologe, spezialisierte Fachberatung, FGI

\footnotetext{
${ }^{1}$ In der spezialisierten Fachpraxis ist umstritten, ob Professionelle den Kontakt mit Chatverläufen und anderen digitalen Zeugnissen grundsätzlich ablehnen sollen. Die Argumente für eine kategorische Ablehnung sind Belastungen, die ein Kontakt nach sich ziehen kann, die mögliche Strafbarkeit des Materials sowie ein beruflich grenzverletzendes Handeln gegen das Einvernehmen von Kindern und Jugendlichen, welche in der Regel nicht zustimmen, dass ihre Chatverläufe Dritten gezeigt werden. Die vorliegende Vignette löst diesen Konflikt nicht. Die Auseinandersetzung mit dem Chat dient der Definition von Kriterien zur fachlichen Einordnung mediatisierter sexualisierter Gewalt.
} 
Insofern ist ergänzend eine fachliche Orientierungsgrundlage geboten, die sich auf die konkreten Handlungen und Interaktionen selbst stützt. Dies gilt ebenso für Problemkonstellationen, in denen nicht Chatverläufe, sondern Fotoaufnahmen (s. Kap. 10) oder das Risiko einer erneuten Konfrontation mit Gewaltaufzeichnungen (s. Kap. 8) eingeordnet werden müssen. Im vorliegenden Situationskontext führt uns dies jedenfalls zu der Frage, welche Hinweise auf mögliche mediatisierte sexualisierte Gewalt der obige Chatauszug enthält.

Vordergründig fällt zweifelsohne die aktive Sexualisierung der Situation durch Dior auf. Aus der Forschung zu Strategien bei Online-Grooming ist bekannt, dass sexualisierte Bewertungen durch gewaltausübende Menschen gezielt zur Desensibilisierung $^{2}$ von Kindern und Jugendlichen eingesetzt werden. Dabei wenden Gewaltausübende sogenanntes Reframing an, also eine sexualisierte Umdeutung oder -rahmung von Situationen (Lorenzo-Dus \& Kinzel, 2019). Sie verwenden unter anderem sexuelle Zweideutigkeiten. Beispielhaft ist im Chatauszug beispielsweise die Verwendung der Ausdrücke „Möpse“ und „Knospen“. Mit der sexualisierten Aufladung verschieben Gewaltausübende die kommunikativen Grenzen, sodass es Gewaltbetroffenen kaum möglich ist, sexualisierte Ausbeutung und Übergriffe als solche zu erkennen bzw. sich dagegen abzugrenzen. Aus der Forschung ist bekannt, dass Gewaltausübende die Sexualisierung in einen Rahmen scheinbarer Vertrautheit und Intimität betten. Diese Scheinnähe lenkt von ihren eigentlichen Motiven ab. Dazu regen sie den Austausch privater Informationen, Gespräche über das gemeinsame Beziehungsverhältnis oder die Beziehung zu Dritten an oder sie loben und schmeicheln Betroffenen (ebd.).

Angenommen, das Verhältnis zu A_Rendelle wäre durch Dior entsprechend beabsichtigt gerahmt, kann A_Rendelles mangelnde Abgrenzung gegen die offensive Sexualisierung nur schwerlich als Einvernehmen bewertet werden. Wir verweisen in diesem Zusammenhang auf das Konzept der informierten Einwilligung. Sie setzt in Anlehnung an Finkelhor (1979) voraus, dass A_Rendelle prinzipiell versteht, was während und als Folge der Beteiligung an sexualisierten Handlungen mit ihr passiert. Zweitens müsste sie die Freiheit haben, die Handlungen auch abzulehnen. Letzteres träfe schon dann nicht zu, wenn sie zum Beispiel Nachteile durch eine Ablehnung sexualisierter Handlungen befürchtete. Es ist nicht auszuschließen, dass sie durch ein gezielt hergestelltes Vertrauensverhältnis bereits befangen ist. Sie wäre dann nicht fähig, sich abzugrenzen oder

\footnotetext{
${ }^{2}$ Desensibilisierung meint Handlungen, die das Ziel verfolgen, die Achtsamkeit von Kindern, Jugendlichen und deren Umfeld für Grenzverletzungen abzuschwächen. Dazu binden Gewaltausübende sexualisierte Anspielungen oder andere Überschreitungen der Intimsphäre schleichend in die alltägliche Kommunikation ein. Letztere werden zur Selbstverständlichkeit und Grenzverletzungen zur Normalität.
} 
in die vorwiegend sexualisierten Motive Diors wissentlich einzuwilligen. Dass sie Diors Forderung eines ,richtigen Fotos“ damit beantwortet, dass sie ihn tief spüren wolle, müsste unter dieser Prämisse als ambivalente Bewältigungsstrategie bewertet werden, um handlungsfähig zu bleiben, und nicht als eine proaktive Beteiligung an sexualisierten Handlungen.

In den Focus Group Interviews wurde dies diskutiert. Dabei hinterfragten Expert*innen auch Hinweise auf eine mögliche reifebedingte Machtasymmetrie zwischen Dior und A_Rendelle. Anlass hierzu gaben Hinweise darauf, dass sich A_Rendelles Brüste womöglich in der Entwicklung befinden („Knospen“) und sie zur Schule gehe. Dior mache dagegen „Feierabend“, was als denkbares Indiz einer Berufstätigkeit und folglich eines Alters- und Reifeunterschieds diskutiert wurde. Jedoch bleibt der Chat diffus und die Einschätzungen der Expert*innen hypothetisch, schließlich gebe es Schüler*innen, die sagten, „ich habe meine Schularbeiten erledigt, ich habe Feierabend.“ (Sozialarbeiter, spezialisierte Fachberatung, FGI)

Zusammenfassend gibt der Chatverlauf Anlass zu gesteigerter Aufmerksamkeit und zweifelsohne Parteilichkeit ${ }^{3}$ mit A_Rendelle. Diese ergeben sich in erster Linie aus der Sexualisierung des Mädchens, dem Rückgriff auf hierarchische Geschlechterrollenerwartungen (,echte Frau“), Verhaltensweisen A_Rendelles, die als ambivalente Bewältigungsstrategie interpretiert werden können, sowie die Erwägung sexualisierten Online-Groomings. Auf alleiniger Basis des Chats ist dennoch nicht auszuschließen, dass A_Rendelle und Dior sich als Gleichberechtigte definieren, die sich in einem Aushandlungsprozess von Beziehungserwartungen befinden. Unter diesen Umständen liegt nicht weniger ein Bedürfniskonflikt zwischen beiden vor. Dior reizt gezielt die Grenzen sexueller Interaktionen aus. Dabei knüpft Dior an eine gesellschaftsstrukturelle Objektifizierung weiblicher Sexualität an, was aus Sicht pädagogischen Gendermainstreamings nicht

\footnotetext{
${ }^{3}$ Das Konzept der Parteilichkeit fußt auf der Annahme, dass soziale Benachteiligung und deren extremste Form, die Gewalt, Ausdruck und Folge gesellschaftlicher Machtasymmetrien sind. Erstens fordert Parteilichkeit Professionelle zunächst zu einer klaren und eindeutigen Position gegenüber Gewalt heraus. Sie geht dabei über eine ausschließliche Fokussierung individueller Benachteiligung hinaus und reflektiert Dynamiken und Strukturen, welche rollenbezogene Mindermacht, Ohnmacht und Gewaltbetroffenheit hervorbringen. Sie reflektiert demnach die politische Dimension von Benachteiligung, ohne hierüber individuelle Unterschiede der Betroffenheit zu pauschalisieren. Zweitens ist Parteilichkeit eng mit der Solidarität (Advocacy) mit Gewaltbetroffenen verbunden. Besonders hervorgehoben sei in diesem Zusammenhang die empathische Perspektivübernahme hin zum Erleben der Gewaltbetroffenen, um Letztere im Sinne einer professionell stellvertretenden Deutung bei der Wahrnehmung ihrer Anliegen, Bedürfnisse und Rechte unterstützen und stärken zu können (Kavemann, 1997; Helfferich \& Kavemann, 2004).
} 
unproblematisch ist. A_Rendelle scheint stärker auf die Gestaltung der Beziehungsintensität fokussiert zu sein. Dies widerspricht jedoch nicht der Möglichkeit eines Einvernehmens des Aushandlungsprozesses. Zudem sehen Kinder und Jugendliche Beziehungen, die vorwiegend medial geführt werden, nicht prinzipiell als problematisch oder dysfunktional an. Darin besteht eine latente Spannung zu beobachtbaren einstellungsbasierten Bewertungsmustern erwachsener Bezugsund Fachpersonen.

\begin{abstract}
„Es ist zu vermuten, dass die Ratsuchende emotional sehr bedürftig war, da sie in dieser eher losen Internetbekanntschaft zumindest zu Beginn eine Art Liebesbeziehung gesehen hat [...]. Es könnte aber auch sein, dass [...] diese Art Beziehungen inzwischen unter Jugendlichen doch sehr normal sind. Dafür würde sprechen, dass uns das in der Arbeit recht häufig begegnet."
\end{abstract}

Falldokumentation 4.2

Junge Menschen bewerten die mediale Gestaltung von Beziehungen durch subjektiv erlebte Exit-Optionen (sich Reaktionen nicht unmittelbar aussetzen zu müssen; sich entziehen zu können) eher als geschützte Kommunikationsform (Scarcelli, 2015). Ergänzende Studien zeigen zwar, dass Jugendliche Freundschaften, die nicht vorwiegend online existieren, nach wie vor als von besonderer Qualität erleben. Grundsätzlich hängt die Ernsthaftigkeit von medial geführten Beziehungen aus Sicht von Kindern und Jugendlichen jedoch nicht davon ab, ob das Gegenüber denselben sozialen Nahraum teilt oder man sich jenseits von Chat, Fotos und Videos bereits getroffen hat (Wood et al., 2016). Daher wäre es unter (sekundär-) präventiven Gesichtspunkten fragwürdig, A_Rendelle mit Verweis auf eine Fernbeziehung vom automatischen Vorliegen einer dysfunktionalen Beziehung oder einer Gewaltbetroffenheit überzeugen zu wollen. In den Handlungsempfehlungen werden Konsequenzen hieraus diskutiert. Zuerst folgt aber ein zweiter Ausschnitt des Chatverlaufs.

\title{
Fallvignette A_Rendelle und Dior
}

\section{Chatverlauf Teil 2, 24. August}

11:05 A_Rendelle: Wieso redest du nicht mehr mit mir? Bist du sauer? 18:06 Dior: Hab doch gesagt: viel zu tun.

18:07 A_Rendelle: Können wir gleich reden? Hab gerade Stress mit meiner Mutter. 
18:14 A_Rendelle: Was ist denn los? Bist du sauer wegen dem Bild oder warum?

18:32 Dior: Vergiss es einfach. Du vertraust mir nicht. Vielleicht lassen wir es besser.

18:32 A_Rendelle: Wieso denn?

22:45 Dior: Machst erst ein auf erfahrene Powerfrau und dann Pustekuchen. Hab mich halt täuschen lassen. Ich dachte, du bist echt.

22:45 A_Rendelle: Was kann ich machen, dass du mir vertraust?? Ich schick dir ein Bild oben ohne. Warte.

22:45 Dior: Laber Rhabarber. Musst du dir schon was Besseres einfallen lassen, wenn du es wieder gut machen willst. Tipp: Reimt sich auf Uschi ;-)

\section{Reflexionsfragen}

- Was nehmen wir im Chat wahr?

- Was löst der Chat in uns aus?

- Wie stehen wir zu den Chattenden und ihrem Verhalten?

- Wie kann die Situation fachlich eingeordnet werden?

\subsection{Reflexionen zur fachlichen Einordnung des Fallbeispiels (Teil 2)}

Seit dem ersten Chat sind zwei Tage vergangen. In A_Rendelles Erleben redet Dior nicht mehr mit ihr. Sie erkundigt sich nach den Gründen. Zunächst wiegelt Dior ab. Erst auf wiederholte Nachfrage führt Dior an, sich habe täuschen zu lassen. Wie schon zuvor (,echte Frau“) wendet Dior Geschlechterrollenerwartungen (,erfahrene Powerfrau“) an und bringt zum Ausdruck, dass A_Rendelle einer angeblich von ihr selbst geweckten Erwartung nicht entspreche. Implizit wertet Dior A_Rendelle in ihrer Geschlechtlichkeit als nicht ,echt“ ab, denn die Vorwürfe mangelnder Authentizität und Täuschung stehen in einem Zusammenhang zur Geschlechterkonstruktion Diors. Außerdem überträgt Dior A_Rendelle die Verantwortung für die Abwertung (,,Machst erst einen auf Powerfrau und dann Pustekuchen"). Anlass ist ein Foto, welches Dior entweder nicht geschickt wurde oder Diors Erwartungen nicht entspricht. Letztere Variante würde zu Diors Forderung nach einem ,richtigen Bild“ im ersten Chatausschnitt passen. In einer 
Hierarchie, in der es ,richtige“ Fotos gibt, müssen folglich auch Fotos existieren, die diesem Anspruch nicht genügen. In den Focus Group Interviews kommentiert dies eine Sexualpädagogin wie folgt:

„Und dann schicke mal ein richtiges Bild. Okay, gibt es auch falsche, sind richtige Bilder, nur die, wo man Knospen erkennen kann. [...] Es gibt es echte Frauen und irgendwie unechte Frauen."

Sozialarbeiterin, Sexualpädagogik, FGI

A_Rendelle erlebt augenscheinlich eine Bringschuld, Vertrauen anhand eines Fotos „oben ohne“ wiederherstellen zu müssen. Dior schlägt dieses Angebot jedoch mittels weiterer Abwertungen („Laber Rhabarber“) aus und suggeriert, zur Wiedergutmachung ein Foto von A_Rendelles Genitalien zu verlangen.

\title{
4.2.1 Welche Hinweise auf mögliche mediatisierte sexualisierte Gewalt enthält der Chat?
}

Zentral für die Einordnung des Chats ist der von Dior geförderte Erwartungsdruck. Dieser beginnt im Umschaltspiel zwischen positiv konnotierter Bewertung und Abwertung A_Rendelles.

\begin{abstract}
„Dann sagt er ihr auch, , also eine echte Frau erkenne ich sofort“ und jetzt, also dreht er das Blatt um und sagt ,ja, okay, du hast auf eine Powerfrau gemacht". Wahrscheinlich bezieht er sich auf den letzten Satz da, was sie da gesagt hat, dass sie ihn tief spüren will, [...] also dreht er das ja halt noch mal um, und das finde ich ja halt so, auch so, so doppelt draufgedrückt. Also zum einen, ja, ich rede jetzt nicht mit dir, weil du mir kein Bild schicken wolltest und dazu bist du auch noch so und so."
\end{abstract}

Psychologin, Forschung sexualisierte Gewalt, FGI

A_Rendelles Entscheidungsspielraum wird augenscheinlich zwischen Bewertung und Abwertung eingeschränkt. Will sie den Erwartungen Diors gerecht werden, muss sie Dior ein Foto ihrer Genitalien schicken. Zugleich fürchtet A_Rendelle offensichtlich einen Rückzug Diors, wie ihre Nachfragen, warum Dior nicht mehr mit ihr rede, andeuten. Auffällig ist auch, dass Dior wiederholt mit einer vergleichsweise großen zeitlichen Verzögerung von mehreren Stunden auf A_Rendelles Fragen antwortet.

Ein hilfreiches Modell zur Einordnung des Geschehens bietet das SensoaFlagge-System (Frans \& Maris, 2018). Das Modell wurde entwickelt, um 
altersadäquate Formen der Sexualität zwischen Kindern bzw. Jugendlichen von sexualisiert grenzverletzendem Verhalten durch Kinder oder Jugendliche zu unterscheiden ${ }^{4}$. Zwecks Anwendung des Systems auf digitale Kontexte ist eine Kenntnis der Altersdifferenz zwischen Dior und A_Rendelle keine notwendige Voraussetzung. Das Modell unterscheidet Faktoren wie eine existierende oder nicht gegebene Gleichwertigkeit von Akteur*innen, die Freiwilligkeit, an sexuellen Handlungen teilzunehmen, eine Kontextangemessenheit von Verhalten oder Hinweise auf eindeutige oder nicht eindeutige Zustimmung in sexuelle Handlungen.

Angewendet auf den zweiten Chatausschnitt gibt es keine eindeutige Zustimmung beider Akteur*innen in die sexualisiert konnotierten Handlungen. A_Rendelles Bereitschaft, Dior ein „Bild oben ohne“ zu schicken, genügt zum wiederholten Male nicht Diors Anforderungen (,Musst du dir schon was Besseres einfallen lassen."). A_Rendelles Reaktion lässt auf eine Ambivalenz, eine Hemmung respektive Widerstände schließen. Eine Freiwilligkeit insbesondere mit Blick auf das Zusenden des Fotos ihrer Genitalien ist daher nicht vorauszusetzen. Dior wendet Überredung und latenten Zwang an. Eine Gleichwertigkeit zwischen A_Rendelle und Dior kann zwar nicht entlang alters- und damit reifebedingter Verhältnisse bestimmt werden. A_Rendelle scheint aber spätestens im zweiten Chatausschnitt situativ stärker in die Beziehung involviert zu sein, wie ihre Besorgnis um einen als solchen erlebten Rückzug Diors oder die Mitteilung vertrauensvoller Informationen (,Stress mit meiner Mutter") nahelegt. Im Gegensatz dazu reagiert Dior wiederholt erst mit mehreren Stunden Verzögerung. Zumindest ein leichtes Ungleichgewicht im Beziehungsverhältnis der beiden kann angenommen werden. Das Sensoa-Flagge-System räumt unter der Kategorie „Gleichwertigkeit“ ein, dass grundsätzliche oder situationsabhängige Machtasymmetrien aufgrund von Reife, Ansehen, Status, Lebenserfahrung oder anderen emotionalen, sozialen und materiellen Ressourcen zahlreiche Beziehungen kennzeichnen. Das System wertet darüber nicht moralisch, sondern fokussiert vor allem Handlungen, in denen sich Machtverhältnisse - wie hier - problematisch auswirken.

Streitbar ist, inwieweit das Verhalten Diors in den Kontext „Chat" und zum Entwicklungsstand A_Rendelles und Diors passt. Zwecks Klärung der Kontextangemessenheit fragt das Sensoa-Flagge-System zunächst prinzipiell, inwieweit ein Verhalten in den Zusammenhang passt, in dem es sich äußert. Wenn ein*e

\footnotetext{
${ }^{4}$ Eine zusammenfassende Darstellung des Konzepts ist online im FORUM Sexualaufklärung unter folgendem Link zu finden: https://forum.sexualaufklaerung.de/ausgaben-ab2010/2018/ausgabe-2/umgang-mit-sexuellem-grenzueberschreitendem-verhalten-von-kin dern-und-jugendlichen/.
} 
Jugendliche*r sich beispielsweise vor einer anderen Person entblößt, entscheidet unter anderem der Kontext über die Angemessenheit des Verhaltens. Es kann einen Unterschied machen, ob das Verhalten im intimen Rahmen eines Privatzimmers oder auf dem Schulhof vor anderen stattfindet. Ähnlich versucht das Flagge-System nach altersangemessenen und altersuntypischen Formen sexualisierter Handlungen zu unterscheiden. Mit Blick auf den obigen Chat geht aus der Forschung hervor, dass Jugendliche das Versenden von selbst hergestellten sexualisierten Abbildungen normalisieren (vgl. Böhm \& Budde, 2020) und dass dies mit Asymmetrien und Ambivalenzen einhergehen kann, wie nachfolgende Äußerung aus den Interviews mit gewaltbetroffenen jungen Menschen zeigen:

\footnotetext{
„Das ist immer schon, glaube ich, das Erste, dass die halt so erste sexuelle Erfahrungen damit [Anm.: Nacktfotos schicken] sammeln, und wenn die Mädchen das dann nicht machen, dass die vielleicht sauer sind, und die Mädchen, weil sie in die verliebt sind, das dann deswegen machen, um sie zu beeindrucken. Das steckt, glaube ich, einfach dahinter.“

Gewaltbetroffene, Interview

„IP: Der hat davor geschrieben so, ,ja, kannst du mir was senden?` Mit senden meinte er natürlich so Nacktbilder.

I: Das weiß man sofort, dass das gemeint ist?

IP: Ja, klar. Ich auf jeden Fall. ,Nein, mache ich nicht, nein!‘ Dann hat der mich so richtig provoziert."

Gewaltbetroffene, Interview
}

Aus fachlicher Perspektive ist die Frage nach der Kontextangemessenheit unter Berücksichtigung des ausgeübten Drucks normativ zu begründen: Kinder oder Jugendliche sollten sich in digitalen Kontexten nicht genötigt fühlen, anderen Nacktfotos von sich zu schicken. Ableiten lässt sich dies aus dem Belastungsrisiko, dem A_Rendelle durch den Kontrollverlust (hier bereits im Moment des Versendens) und die zu erwartende Verletzung ihrer Intimsphäre ausgesetzt ist. Dior sollte zudem widerständische Signale A_Rendelles wahrnehmen können, schließlich beziehen sich Diors Vorwürfe auf eine Nichtentsprechung A_Rendelles (,Machst erst einen auf Powerfrau und dann Pustekuchen“). Diors Verhalten ist dahingehend absichtsvoll. Es missachtet A_Rendelles Widerstände und verstärkt ihre Ambivalenzen. Spätestens die Aufforderung, ein Foto ihrer Genitalien zu schicken, ist daher eine beabsichtigte sexualisierte Grenzverletzung gegenüber A_Rendelle, also ein sexualisierter Übergriff und somit mediatisierte sexualisierte Gewalt (Hipp et al., 2017). 
In den Focus Group Interviews des HUMAN-Projekts gehen die Deutungen, wie der Chat auf A_Rendelle wirken könnte, weiter. Ein spezialisierter Fachberater für Kontexte sexualisierter Gewalt stellt folgende These auf:

„Sie fragt ,wieso denn“ und er antwortet vier Stunden lang gar nichts und sie denkt die ganze Zeit alle möglichen Begründungen durch und ist dann schon sozusagen weichgekocht, wenn er mit der neuen Anfrage kommt. Für sie ist es ja auch eine Lektion, ,hätte ich gleich ein Bild oben ohne geschickt, dann müsste ich jetzt nicht meine Muschi fotografieren. Weil ich mich damals geweigert habe, muss ich jetzt sogar noch einen weitergehen, das heißt, beim nächsten Mal mache ich gleich, was er von mir will, damit es keinen Stress mit ihm auch noch gibt.““

Soziologe, spezialisierte Fachberatung, FGI

Die Deutung knüpft an das Konzept der im Zusammenhang von OnlineGrooming erforschten Täter*innenstrategie sogenannter Reverse Psychology an. Ziel der Strategie ist es, die betroffene Person zu sexualisiert ausbeuterischen Handlungen herauszufordern (Lorenzo-Dus \& Kinzel, 2019). Die gewaltausübende Person verhält sich dabei gegenteilig zu ihren tatsächlichen Motiven. Zum Beispiel täuscht sie vor, sie habe das Interesse an ihrem Gegenüber oder sexuellen Handlungen mit demselben verloren. Die Forschung zu Online-Grooming legt zudem nahe, dass Täter*innen oft positiv konnotiert mit Betroffenen kommunizieren, sie loben und umschmeicheln. Umso schwerer wiegt dann ein strategischer Entzug (Broome et al., 2018). Ein Ergebnis der internationalen Forschung mit Gewaltbetroffenen von sexualisiertem Online-Grooming ist passend hierzu, dass sie besonders den Kontaktverlust zu der gewaltausübenden Person fürchten, wenn ein erstes Beziehungsverhältnis besteht (Whittle et al., 2014).

Ein Begünstigungsfaktor dafür wurde von Expert*innen in den Focus Group Interviews unter dem Stichwort der strukturellen Vulnerabilität junger Menschen verhandelt:

„Wenn wir jetzt hier bei den Mädchen sind, suchen Mädchen ja auch nach einer Erwachsenenidentität. Und dann sind da/ und dann kommt da jemand, der gibt ihnen genau das, Bestätigung in der Rolle, in die sie sich gerade reinfinden möchten. Und das ist natürlich auch etwas total Entlastendes, das ungefragt projiziert zu bekommen. Naja, du bist erwachsen, du bist eine Frau, du bist gleichberechtigt, du bist eine potenzielle Partnerin für mich und gleichz.../ Also, ich glaube, das ist nicht nur einfach dieser Punkt, dass es die sozial Vulnerablen sind, sondern [Anm. junge Menschen] sind einfach strukturell vulnerabel.“

Psychologin, Täter*innentherapie, FGI 
$\mathrm{Zu}$ ähnlichen Schlüssen kam die Sexualwissenschaftlerin Schönfelder bereits in den 1960er Jahren, als sie feststellte, dass eine Verwicklung von Kindern und Jugendlichen in sexualisiert gewalttätige Beziehungen nicht als sexuelle Neugier zu missverstehen sei: „Wesentlich ist vielmehr die vom Partner vollzogene Überhöhung, zu einem Zeitpunkt schon als Frau zu gelten, in dem die übrigen Erwachsenen ihre tabuisierte Welt dem Kind noch verschließen." (Schönfelder, 1965, S. 111).

\subsubsection{Wann ist die Bezeichnung Täter*in angemessen?}

Inwieweit Dior als Täter*in bezeichnet werden sollte, kann mit dem Chatverlauf nicht endgültig beantwortet werden. Die Bewertung hängt erneut davon $a b$, ob Dior ein Kind, ein*e Jugendliche*r oder ein*e Erwachsene*r ist, wie groß der Alters- und Reifeunterschied zu A_Rendelle ist, inwieweit Dior den Reifeunterschied im digitalen Kontext einschätzen kann, welche Absichten Dior weiterhin verfolgt, zu welchem Zweck eventuelle Aufnahmen von A_Rendelles Genitalien angefertigt werden und was damit geschieht, wie feststehend Diors übergriffiges Verhalten bereits ist, ob Dior für (pädagogische) Interventionen hinsichtlich des grenzverletzenden Verhaltens empfänglich ist, also einsehen kann, dass es sich um eine Verletzung A_Rendelles handelt, und ob Diors Verhalten veränderbar ist.

Angenommen Dior und A_Rendelle wären beide vierzehn Jahre alt, bleibt Diors Verhalten zwar nicht minder eine beabsichtigte sexualisierte Grenzverletzung. Dior auf ausschließlicher Basis des Chatverlaufs das Etikett „Täter*in“ zu verleihen, wäre jedoch unangemessen und kontraproduktiv. Gerade unter täter*innenpräventiven Gesichtspunkten, die auf eine Verhaltenseinsicht und -änderung zielen, ist auf die voreilige Stigmatisierung junger Menschen zu verzichten. Eine Reduzierung auf sexualisierte Übergriffigkeit wirkt sich hemmend auf Entwicklungspotenziale aus. Vielmehr sollte pädagogisch oder therapeutisch bei dem inadäquaten, zu ändernden Verhalten angesetzt werden. Eine gewaltausübende Person, die sich als Mensch angenommen fühlt, hat bessere Chancen, ihr Verhalten zu verändern. (Kettritz, 2018).

Ist der Alters- und Reifeunterschied zwischen A_Rendelle dagegen größer, A_Rendelle womöglich ein Kind und Dior ein*e Jugendliche*r oder Erwachsene*r, der*die wiederholt strategisch vorgeht, um A_Rendelle durch die Herstellung von Missbrauchsabbildungen oder „kinderpornografischen Inhalten“ im strafrechtlichen Sinne zu missbrauchen, oder Missbrauchsabbildungen sogar verbreitet, trifft die Bezeichnung „Täter*in“ zu. Neben der fachlichen Einschätzung 
ist vor dem Hintergrund sogenannter „Opfergerechtigkeit“ oder besser Gewaltsensibilität zu klären, welchen Zweck, eine Etikettierung Diors als Täter*in im Rahmen einer möglichen Unterstützung A_Rendelles erfüllt. Ob also eine Entlastung A_Rendelles hierdurch zu erwarten ist, oder sie an einem anderen Punkt der Verarbeitung ihrer Verletzungserfahrungen steht und die Labels „Täter*in“ bzw. „Opfer" sogar abwehrt.

\subsection{Handlungsempfehlungen}

Aufgrund des fehlenden Fallkontexts sind die Handlungsempfehlungen der vorliegenden Vignette eher als Maxime (Prinzipien) zu verstehen. Konkrete Handlungsschritte werden verstärkt in den nachfolgenden Falldiskussionen thematisiert. Die Maxime können als Anhaltspunkte jener einstellungs- und wertebasierten Muster verstanden werden, die unsere Haltung im Umgang mit Gewaltbetroffenen definieren.

Wir halten zunächst nochmals fest:

A_Rendelle erlebt eine beabsichtigte, sexualisierte Grenzverletzung bzw. einen sexualisierten Übergriff. Die Einordnung als sexualisierte Gewalt ergibt sich aus der Sexualisierung der Situation, einen durch Druck und Verunsicherung eingeschränkten Handlungsspielraum (Machtungleichgewicht) sowie Diors Versuche, A_Rendelles Widerstand, ein Nacktfoto zu schicken, aktiv zu überwinden, das heißt ihre Einwilligungsfähigkeit zu umgehen. Kriterien zur Gewalteinordnung der Expert*innen aus den Focus Group Interviews sind:

- Aufbau von Abhängigkeit und Druck,

- Anknüpfen an hierarchische Rollenerwartungen („echte Frau“) und Beziehungsmuster, das heißt die Instrumentalisierung eines Machtgefälles: „Dass Sexualität instrumentalisiert wird, um Macht auszuüben und letztendlich auch eine Form von Unterdrückung, die sich dann natürlich patriarchalischem Muster anschließt oder es nutzt." (Soziologe, spezialisierte Fachberatung, FGI)

- Aushebeln der Selbstbestimmung durch eine Verschleierung eigener Motivationen: „Kann der andere sexuell selbstbestimmt handeln und liegt eine unterschiedliche Motivation vor? Und ich habe das Gefühl, dass das hier nicht der Fall ist, es gibt unterschiedliche Motivationen, die sexuelle Selbstbestimmung, Integrität, das finde ich, wird an der Stelle nicht gewahrt.“ (Psychologin, Täter*innentherapie, FGI)

- Gezielter Missbrauch von Vertrauen: „Sehen wir eigentlich das, was das Verletzende ist für die Betroffenen wirklich im aktuellen Diskurs. [...] Die 
Beziehung fühlt sich so echt an, aber dann ist der Vertrauensmissbrauch genau analog zu/ wenn das meine Mutter machen würde, also genauso eng. Und dann ist das aber auch genauso wirksam und manchmal vielleicht das, was das Verletzendste ist.“ (Soziologe, spezialisierte Fachberatung, FGI)

An A_Rendelles Betroffenheit und Unterstützung richtet sich der Fokus der Handlungsprinzipien aus. Wir verfolgen den Ansatz der Parteilichkeit weiter, nachdem wir Gewalt gegen A_Rendelle feststellen konnten. Das Konzept der Parteilichkeit reflektiert erstens Dynamiken und Strukturen, die rollenbezogene Mindermacht, Ohnmacht und Gewaltbetroffenheit hervorbringen. In unserem Fall ist dies der Entsprechungsdruck, welcher erzeugt wird durch Geschlechterbezüge, die Reinszenierung hierarchischer Beziehungsmuster und damit verbundene sexuelle (An-)Forderungen. Die Verantwortung für die Gewalt liegt bei Dior. A_Rendelles Ambivalenz und etwaige Schuld- und Schamgefühle müssen in einer Wechselwirkung zur Gewalt betrachtet werden. Zweitens ist Parteilichkeit mit der Solidarität (Advocacy) mit Gewaltbetroffenen verbunden (Kavemann, 1997; Helfferich \& Kavemann, 2004). Dazu gehört eine empathische Perspektivübernahme, um Gewaltbetroffene bei der Wahrnehmung ihrer Anliegen, Bedürfnisse und Rechte unterstützen und stärken zu können. Perspektivübernahme bedeutet in diesem Zusammenhang nicht, A_Rendelles Wahrnehmung einer objektiven Realität gleichzusetzen, sondern ihr subjektives Erleben, ihre Belastungen, Verletzungen, Bedürfnisse und Lösungsansätze als Hilfeauftrag anzuerkennen. Das von dieser Perspektivübernahme ausgehende Zugeständnis konterkariert die erlebte Manipulation, den Vertrauens- und Kontrollverlust sowie mögliche Zweifel an ihrer eigenen Wahrnehmung. Es ist als ermutigender Appell zur Selbstbestimmung gewaltbetroffener junger Menschen zu verstehen.

\subsubsection{Bei der Lebenswirklichkeit von Adressat*innen ansetzen}

Wir wissen nicht, wie A_Rendelle die Situation im Detail bewertet, geschweige denn, ob sie Diors Verhalten als Gewalt definiert. Um sich ihrer Perspektive anzunähern, müsste A_Rendelle angesprochen und selbst gefragt werden. Eine Annäherung an ihre Lebenswirklichkeit ist nur im Austausch möglich. Zur Verdeutlichung dieses Zugangs stellen wir die These auf, dass A_Rendelle das Verhältnis zu Dior als bedeutungsvoll und Diors Rückzug als beängstigend erlebt. Einen Kontaktabbruch fürchtet sie ausreichend, um Forderungen, denen sie nicht 
unmittelbar entsprochen hat, jetzt nachzukommen. Gleichzeitig entsteht oder verstärkt sich durch die Angst vor dem Kontaktverlust ein innerer Konflikt. Das Verhältnis zu Dior entwickelt sich nicht so, wie A_Rendelle sich dies wünscht.

Aus parteilicher Perspektive ist A_Rendelle bezüglich ihrer eigenen Vorstellungen von Beziehungsgestaltung ernst zu nehmen. Handlungsleitend sind sodann folgende Fragen:

- Was wünscht sie sich?

- Welche Bedürfnisse hat sie?

- Inwieweit erfüllen sich ihre Bedürfnisse im Verhältnis zu Dior?

- Was erfüllt sich nicht?

- Wie geht es ihr damit?

- Welche Verhaltensweisen Diors enttäuschen sie?

Im Sinne kritisch-emanzipatorischer Pädagogik ist sie bei der Wahrnehmung ihrer Bedürfnisse zu bestärken.

\subsubsection{Adressat*innen Resonanz anbieten}

Da der Chatverlauf als Gewalt eingeordnet wird, besteht Grund zu der Annahme, dass A_Rendelle womöglich weiterhin sexualisierter Gewalt ausgesetzt ist. Angesichts dessen stellt der Austausch mit dem Mädchen aus der Perspektive Helfender nicht ausschließlich ein Beziehungsgespräch, sondern ein Hilfegespräch dar. Aus parteilicher Perspektive ergibt sich hieraus ein Konflikt, nämlich dem Mädchen gegenüber transparent sein und gegebenenfalls sogar Interventionen begründen zu müssen, gleichzeitig aber ihre Deutungen zu respektieren und sie nicht davon überzeugen zu wollen, dass sie „Opfer“ geworden ist. Aus den Interviews geht hervor, dass jugendliche Betroffene auch rückwirkend von gewaltausübenden Personen als Freund*innen sprechen, wenn die Gewalt in eine positiv bewertete Beziehungsgestaltung gebettet war. Enttäuscht sind sie eher davon, dass ihnen jemand eine Beziehung vorgetäuscht hat, in der gelogen, sexualisierte Handlungsmotive verschleiert und ein Interesse an der eigenen Person nur so lange aufgebracht wurde, wie die gewaltausübende Person bekam, was sie wollte. Die Konfrontation mit dem Label Gewalt bzw. Überzeugungsversuche, dass hier Gewalt vorliege, kann problematisch sein, wenn Betroffene an einem anderen Punkt der Verarbeitung stehen. Die im HUMAN-Projekt analysierten Falldokumentationen zeigen, dass solcherlei Definitionsmachtkonflikte zwischen Gewaltbetroffenen und Helfenden häufig zu Beratungsabbrüchen führen. 
Fachliche Begründungen des Hilfeanlasses sind deshalb als Resonanzangebot und nicht als Überzeugungsversuche zu gestalten. Dabei kann in einer Sprache, die an den Verarbeitungsstand A_Rendelles anknüpft, zwischen der gewaltausübenden Person, dem gewalttätigen Verhalten und der Bewertung des Verhaltens unterschieden werden. Es ist denkbar zu erklären, weshalb man als Beratende*r das Verhalten Diors als Druckaufbau bzw. Ausnutzung wahrnimmt. Sinnvoller als normative Äußerungen (,Du musst verstehen, dass das Gewalt ist.“) erscheinen authentische Ich-Botschaften, da sie ein Kontaktangebot beinhalten: „Wenn ich mir vorstelle, dass jemand sich mir gegenüber so verhält, würde es mir [so] gehen." Alternativen, A_Rendelle einen Perspektivwechsel auf Diors Verhalten zu ermöglichen, sind zirkuläre Fragen: „Wie würde es Dir gehen/ was würdest Du denken/ was würdest Du tun, wenn Dir eine Freundin erzählen würde, dass sich ihr*e Freund*in ihr gegenüber so verhält?“*

Die Intervention setzt ein verlässliches Beratungsverhältnis zwischen der Adressatin und der*den*dem Helfenden voraus. Dann kann A_Rendelle das Hilfemotiv verstehen, mit ihren eigenen Erfahrungen abgleichen und erlebt womöglich eine positive Stärkung hinsichtlich eigener Zweifel und Ambivalenzen, ohne personenumfassende Stigmata annehmen oder abwehren zu müssen.

\subsubsection{Selbstwirksamkeit stärken}

Eine Selbstwirksamkeitsstärkung A_Rendelles beginnt im Moment des Zugeständnisses durch die Perspektivübernahme und die Arbeit an ihren Bedürfnissen. Die Falldokumentationen zeigen zudem, dass spezialisierte Fachpraktiker*innen Betroffene nach widerfahrener Gewalt bei einer selbstbestimmten Nutzung digitaler Medien unterstützen wollen, die als geschützt erlebt wird. Da es sich hierbei um ein (tertiär-)präventives Anliegen handelt, setzt die Arbeit an der Mediennutzung vorherige Aufarbeitungsschritte voraus, ansonsten besteht das Risiko, dass insbesondere Fragen der Art „Wie kannst du dich im Internet schützen?“ als Vorwurf an ein vorheriges Versäumnis verstanden werden. Sie schwächen Gewaltbetroffene.

Hilfreich ist deswegen vielmehr eine Arbeit entlang von A_Rendelles Ambivalenzen. Sie erlaubt die Auseinandersetzung mit der Frage, weshalb es so schwierig ist, eigene Grenzen wahrzunehmen, wenn eine andere Person, der man vertraut, Druck aufbaut und diesen durch Projektionen in digitalen Kontexten verstärkt. Die Arbeit an A_Rendelles Ambivalenzen verknüpft eine Abgrenzung gegenüber Diors übergriffigem Verhalten mit der Förderung von A_Rendelles 
Selbstwirksamkeit. Die Ambivalenz des Mädchens beinhaltet emotionale Widersprüche und Widerstände, die zwar nicht eindeutig, aber spürbar sind. Sie können als leibliche Signale einer Schräglage verstanden werden, die auf A_Rendelles Grenzen verweisen. Selbstwirksamkeit zu stärken bedeutet daher, Ambivalenzen herauszuarbeiten, Achtsamkeit für Ambivalenzen zu fördern, eigene Bedürfnisse hinter den Ambivalenzen zu erkennen, diese in ein Verhältnis zu den Auslösern der Ambivalenz setzen und Möglichkeiten oder Alternativen zur Verhandlung bzw. Durchsetzung der eigenen Bedürfnisse zu entwickeln. Freilich handelt es sich hierbei um einen aufwendigen Prozess, der perspektivisch ist und professionelle Krisenintervention zum Schutz von Kindern und Jugendlichen nicht ersetzt.

\subsubsection{Strafanzeige höchstens fallabhängig abwägen}

Eine Strafanzeige auf Basis der Chatausschnitte ist nicht ohne weiteres zielführend. Erstens genügen die gegebenen Situationsmerkmale nicht, um Diors Verhalten als strafwürdig zu identifizieren. Die Bewertung der Strafwürdigkeit ändert sich zwar in dem Augenblick, in dem A_Rendelle unter 14 Jahren alt wäre. Dann kann Diors Verhalten als sexueller Kindesmissbrauch nach § 176 Abs. 4 Nr. 3 StGB bewertet werden. Die Aufforderung, ein Foto der Genitalien zu schicken, ist hierbei entscheidend. Allerdings kennen wir A_Rendelles Alter nicht. Zudem ist nicht geklärt, wie A_Rendelle zu einem Strafprozess steht, welche Unterstützung sie aus ihrem sozialen Umfeld erfährt, welchen Belastungen sie durch das Verfahren ausgesetzt wäre, wie ihre Vernehmungsfähigkeit einzustufen ist und welches Ziel mit einer Strafanzeige verfolgt werden soll. Zu berücksichtigen ist dabei, dass einige Falldokumentationen des HUMAN-Projekts Hinweise darauf enthalten, dass Betroffenen im Strafverfahren eine Mitverantwortung oder -schuld unterstellt wird, wie das nachfolgende Zitat aus einem Fall schweren sexuellen Kindesmissbrauchs (Vergewaltigung) durch einen Erwachsenen an einer 12-Jährigen vergegenwärtigt:

„Der Täter ist soweit geständig. Die Staatsanwaltschaft schreibt dem Mädchen allerdings eine Mitschuld zu.“

Falldokumentation 4.3

Im zitierten Fall wurde zum Nachteil des Mädchens ausgelegt, dass sie in Treffen, in denen es zur Vergewaltigung kam, eingewilligt hatte. Eine ähnlich absurde Argumentation könnte um A_Rendelles Äußerung, sie wolle Dior richtig 
tief spüren, aufgebaut werden. Eine Strafanzeige ist daher einer Unterstützung A_Rendelles nicht gleichzusetzen. Sie ist ein ultimatives Instrument zum Schutz Gewaltbetroffener, sofern sie Aussicht auf Erfolg hat. Zuvor sollte aber unter Einbezug mehrerer Fachkräfte eine Abklärung der Zweckdienlichkeit im Verhältnis zur erwarteten Belastung des Mädchens stattfinden. Eine hilfreiche Kritik zum Thema der Strafanzeige legen Kliemann und Fegert (2015) vor.

Sofern Fachkräfte im Kontext ihrer beruflichen Tätigkeit Kenntnis davon erhalten, dass ein Kind in einen vergleichbaren Chat verwickelt ist, sind kollegiale Fachberatung, die Hinzuziehung einer insoweit erfahrenen Fachkraft oder einer spezialisierten Fachberatungsstelle empfohlen. Nur unter Berücksichtigung der Lebenssituation und Perspektive des Mädchens, ihres Umfeldes und der Aussicht, die ein Strafverfahren hat, kann adäquat hierüber entschieden werden. Angenommen A_Rendelle und Dior sind Jugendliche und A_Rendelle sieht keinen Anlass zu einer Bestrafung Diors, die voraussichtlich mit weiteren Übergriffen wie zum Beispiel einer Verbreitung möglicher sexuell expliziter Fotos begründet werden müsste, erscheint eine Strafanzeige nicht sinnvoll.

\subsubsection{Gedanken zur Täter*innenprävention}

Der Schwerpunkt des HUMAN-Projekts liegt auf Interventions- und Unterstützungsmöglichkeiten für Gewaltbetroffene. Deswegen wird auf einen fachlichen Umgang mit Dior nur kurz eingegangen. Zuerst muss festgestellt werden, wie systematisch und gefestigt Diors Verhalten (u. a. in anderen Kontexten) zu bewerten sei. Angenommen Dior wäre ein Kind oder ein*e Jugendliche*r, welche*r*s erste Anzeichen von Übergriffigkeit zeigt, mögen täter*innenpräventive pädagogische Interventionen, in denen erörtert wird, dass das Verhalten verletzend ist, Diors Motive und ihre Ursachen reflektiert, eigene Belastungen beleuchtet und Handlungsalternativen entwickelt werden, Verhaltensänderungen noch anregen. Eine valide Einschätzung sollte bereits von einer im Umgang mit sexualisiert übergriffigen jungen Menschen spezialisierten Person vorgenommen werden.

Wird Diors Verhalten dagegen als strategisch angebahnt bewertet, benötigt Dior andere Formen der Unterstützung (ambulant, teilstationär oder stationär). Dies gilt, um weiterer schwerer Gewalt vorzubeugen. Die Voraussetzung einiger Hilfeeinrichtungen für sexualisiert übergriffige Jugendliche setzen eine Strafanzeige oder eine Meldung beim Jugendamt voraus, um die Wahrscheinlichkeit für Therapie- und Beratungsabbrüche zu reduzieren. Grundlagen der Arbeit mit sexualisiert übergriffigen Jugendlichen erörtert Kettritz (2014). Komplexer gestaltet sich der Fall, wenn Dior erwachsen ist. Das Feld ist stark 
medizinisch-psychiatrisch orientiert. Bereits in der Diagnostik existiert eine große Heterogenität der Zugänge (Beier, 2018). Zudem stehen sich die Schulen der Sexualmedizin und Sexualforensik gegenüber, die den Grundsatz der Opfergerechtigkeit in Täter*innentherapien sehr divers auslegen. Hiervon zu unterscheiden sind Ansätze der opfergerechten Täter*innenarbeit. Informationen hierzu erteilt die Deutsche Gesellschaft für Prävention und Intervention für Kindesmisshandlung, -vernachlässigung und sexualisierte Gewalt e. V. (DGfPI e. V.).

\section{Literatur}

Albrecht, R. (2017). Beratungskompetenz in der Sozialen Arbeit. KONTEXT, 48(1), 45-64. https://doi.org/10.13109/kont.2017.48.1.45.

Beier, K. M. (Hrsg.). (2018). Pädophilie, Hebephilie und sexueller Kindesmissbrauch: Die Berliner Dissexualitätstherapie. Springer VS.

Böhm, M., \& Budde, J. (2020). Selbstbestimmt, konsensuell und wechselseitig? Perspektiven von Schüler*innen auf sexuelle Kommunikation mittels digitaler Medien am Beispiel Sexting. Kindesmisshandlung und -vernachlässigung, 23(1), 6-17.

Broome, L. J., Izura, C., \& Davies, J. (2018). Linguistic characteristics of online grooming ,Relationships'. University of Swansea.

Finkelhor, D. (1979). What's wrong with sex between adults and children? Ethics and the problem of sexual abuse. The American Journal of Orthopsychiatry, 49(4), 692-697. https://doi.org/10.1111/j.1939-0025.1979.tb02654.x.

Frans, E., \& Maris, S. (2018). Umgang mit sexuellem (grenzüberschreitendem) Verhalten von Kindern und Jugendlichen. FORUM Sexualaufklärung und Familienplanung, 2, 43-48.

Helfferich, C., \& Kavemann, B. (2004). Wissenschaftliche Untersuchung zur Situation von Frauen und zum Beratungsangebot nach einem Platzverweis bei häuslicher Gewalt: „Platzverweis - Beratung und Hilfen “. Forschungsprojekt.

Hipp, T. N., Bellis, A. L., Goodnight, B. L., Brennan, C. L., Swartout, K. M., \& Cook, S. L. (2017). Justifying sexual assault: Anonymous perpetrators speak out online. Psychology of Violence, 7(1), 82-90. https://doi.org/10.1037/a0039998.

Kärgel, K., \& Vobbe, F. (2020). Mediatisierte Gewalt - Diffusion - Transzendenz: Erscheinungsformen und Herausforderungen sexualisierter Gewalt mit digitalem Medieneinsatz. Kindesmisshandlung und -vernachlässigung, 23(1), 30-43.

Kavemann, B. (1997). Zwischen Politik und Professionalität: Das Konzept der Parteilichkeit. In Institut Frau und Gesellschaft. (Hrsg.), Parteilichkeit und Solidarität: Praxiserfahrungen und Streitfragen zur Gewalt im Geschlechterverhältnis. Kleine Verlag.

Kettritz, T. (2014). Grenzverletzende Kinder und Jugendliche - verletzte Menschen mit verletzten Grenzen?! Traumapädagogische Arbeit mit sexuell übergriffigen Kindern und Jugendlichen. In P. Mosser \& H.-J. Lenz (Hrsg.), Sexualisierte Gewalt gegen Jungen: Prävention und Intervention: Ein Handbuch für die Praxis (S. 211-261). Springer VS. 
Kettritz, T. (2018). Pädagogische und therapeutische Arbeit mit Kindern und Jugendlichen mit sexualisiert übergriffigem Verhalten. In A. Retkowski, A. Treibel, \& E. Tuider (Hrsg.), Handbuch sexualisierte Gewalt und pädagogische Kontexte: Theorie, Forschung, Praxis (S. 661-669). Beltz Juventa.

Kliemann, A., \& Fegert, J. (2015). Leitlinie der AG II des Runden Tisches zur Einschaltung der Strafverfolgungsbehörden. In J. Fegert \& M. Wolff (Hrsg.), Kompendium „Sexueller Missbrauch in Institutionen“. Entstehungsbedingungen, Prävention und Intervention (S. 486-500). Beltz Juventa.

Orbach, S. (2013). The commercialisation of girls' bodies. In J. Wild (Hrsg.), Exploiting childhood (S. 110-119). Jessica Kingsley Publishing.

Lorenzo-Dus, N., \& Kinzel, A. (2019). ,So is your mom as cute as you?‘: Examining patterns of language use in online sexual grooming of children. Journal of Corpora and Discourse Studies, 2(1), 1-30. https://doi.org/10.18573/jcads.31.

Scarcelli, C. M. (2015). Adolescents, digital media and romantic relationships. Interdisciplinary Journal of Family Studies, 20(2), 36-52.

Schönfelder, T. (1965). Die Initiative des Opfers. Beiträge zur Sexualforschung, 33, 109-115.

Whittle, H. C., Hamilton-Giachritsis, C. E., \& Beech, A. R. (2014). „Under His Spell“: Victims' perspectives of being groomed online. Social Sciences, 3, 404-426.

Wood, M. A., Bukowski, W. A., \& Lis, E. (2016). The digital self: How social media serves as a setting that shapes youth's emotional experiences. Adolescent Research Review, 1, $163-173$.

Open Access Dieses Kapitel wird unter der Creative Commons Namensnennung 4.0 International Lizenz (http://creativecommons.org/licenses/by/4.0/deed.de) veröffentlicht, welche die Nutzung, Vervielfältigung, Bearbeitung, Verbreitung und Wiedergabe in jeglichem Medium und Format erlaubt, sofern Sie den/die ursprünglichen Autor(en) und die Quelle ordnungsgemäß nennen, einen Link zur Creative Commons Lizenz beifügen und angeben, ob Änderungen vorgenommen wurden.

Die in diesem Kapitel enthaltenen Bilder und sonstiges Drittmaterial unterliegen ebenfalls der genannten Creative Commons Lizenz, sofern sich aus der Abbildungslegende nichts anderes ergibt. Sofern das betreffende Material nicht unter der genannten Creative Commons Lizenz steht und die betreffende Handlung nicht nach gesetzlichen Vorschriften erlaubt ist, ist für die oben aufgeführten Weiterverwendungen des Materials die Einwilligung des jeweiligen Rechteinhabers einzuholen.

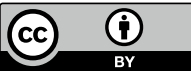

